\title{
Freqüencia de publicações sobre a asma em periódicos de enfermagem indexados em bases de dados brasileiros: uma revisão da literatura
}

\author{
Frequency of publication of asthma studies in Nursing journals indexed in Brazilian databases: a \\ literature review
}

Frecuencia de publicaciones sobre asma en periódicos de enfermería indexados en las bases de datos brasileñas: una revisión de la literatura

\section{Luisa Helena de Oliveira Lima', Emanuel Moura Gomes², Violante Augusta Batista Braga ${ }^{3}$}

\section{RESUMO}

Objetivo. Analisar a freqüência das publicações sobre asma nos periódicos de enfermagem estrangeiros, presentes nos índices de referências informatizados da Coordenação de Aperfeiçoamento de Pessoal de Nível Superior e nacionais indexados em bases de dados informatizadas, considerando-se origem, índice de referência, periódico, ano de publicação, método utilizado, tipo de publicação e objeto de estudo. Métodos. Os dados foram coletados através de formulário, utilizando-se os descritores de assunto asthma e asma para busca das produções. Resultados. Foram identificados 56 artigos estrangeiros e três nacionais nos anos de 1994 a 2004. Os estudos sobre asma aparecem com maior freqüência nos anos de 2002 (12 estrangeiros e 1 nacional) e 2004 (14 estrangeiros). Conclusões. Este trabalho revelou a necessidade de maior produção e/ou divulgação do conhecimento produzido, principalmente em publicações brasileiras.

Descritores: Asma/enfermagem; Publicações científicas e técnicas

\begin{abstract}
Purpose: To describe the frequency of asthma publications in national and international nursing journals indexed in the "Coordenação de Aperfeiçoamento de Pessoal de Nível Superior" or other Brazilian databases. Methods: Database searches were limited to study origin, reference index, journal type, publication year, study methodology, publication type, and object of study "Asthma" or "Asma". Data were collected through a form designed for this study. Results: Databases search retrieved 56 foreign and 3 Brazilian studies conducted between 1994 and 2004. Studies were published more frequently in the years of 2002 (12 foreign and 1 Brazilian) and 2004 (14 foreign). Conclusions: It's necessary further research in Asthma must be conducted and published by Brazilian researchers.
\end{abstract}

Keywords: Asthma/nursing; Scientific and technical publications

\section{RESUMEN}

Objetivo. Analizar la frecuencia de las publicaciones sobre el asma en las revistas de enfermería extranjeras, presentes en los índices de referencias informatizadas de la Coordinación de Perfeccionamiento de Personal de Nivel Superior y nacionales indexados en bases de datos informatizadas, considerándose origen, índice de referencia, periódico, año de publicación, método utilizado, tipo de publicación y objeto de estudio. Métodos. Los datos fueron recolectados a través de un formulario, utilizándose los descriptores del asunto asthma y asma para la búsqueda de las producciones. Resultados. Fueron identificados 56 artículos extranjeros y tres nacionales en los años de 1994 al 2004 . Los estudios sobre asma aparecen con mayor frecuencia en los años 2002 (12 extranjeros y 1 nacional) y 2004 (14 extranjeros). Conclusiones. Este trabajo reveló la necesidad de una mayor producción y/o divulgación del conocimiento producido, principalmente en publicaciones brasileñas.

Descriptores: Asma/enfermería; Publicaciones científicas y técnicas

\footnotetext{
${ }^{1}$ Mestre em Enfermagem pela Universidade Federal do Ceará; Professora da Faculdade Católica Rainha do Sertão - Quixadá (CE), Brasil.

${ }^{2}$ Enfermeiro do Centro de Atenção Psicossocial - Fortaleza (CE), Brasil.

${ }^{3}$ Doutora em Enfermagem; Professora Adjunta do Departamento de Enfermagem da Universidade Federal do Ceará - UFC - Fortaleza (CE), Brasil.
} 


\section{INTRODUÇÃO}

Asma é uma doença inflamatória crônica caracterizada por hiper-responsividade das vias aéreas inferiores e por limitação variável ao fluxo aéreo, reversível espontaneamente ou com tratamento, manifestando-se clinicamente por episódios recorrentes de sibilância, dispnéia, aperto no peito e tosse, particularmente à noite e pela manhã ao despertar. Resulta de uma interação entre genética, exposição ambiental e outros fatores específicos que levam ao desenvolvimento e manutenção dos sintomas ${ }^{(1)}$.

A asma é considerada a mais importante alergia respiratória, tendo em vista suas sérias implicações físicas, sociais e econômicas, as quais determinam sofrimento tanto ao indivíduo portador como a seus familiares.

Cerca de $10 \%$ da população mundial é afetada pela asma, configurando-a como um sério problema de Saúde Pública $^{(2)}$. No Brasil, segundo o DATASUS do Ministério da Saúde, ocorreram cerca de 330.000 internações por asma no ano de 2004, constituindo-se na terceira ou quarta causa de hospitalizações pelo Sistema Único de Saúde (SUS). Destas, a região Nordeste é responsável por 43,6\%. Com relação à esta região, o Ceará é responsável por $15 \%$ das internações ${ }^{(-, 4)}$.

De acordo com a fase 3 do International Study of Asthma and Allergies in Childhood (ISAAC), que foi realizado em 21 centros de 20 cidades brasileiras, distribuídas nas cinco regiões do país, foi observada a prevalência média $24,3 \%$ de asma ativa entre escolares de 6 a 7 anos e 19,0\% entre adolescentes de 13 a 14 anos. As taxas de prevalência de asma e doenças alérgicas mais elevadas foram observadas nos centros das Regiões Norte e Nordeste, exceção feita à de asma, que também foi observada na Região Sul ${ }^{(5)}$.

Apesar do aumento no número de estudos sobre asma, tem-se averiguado que sua morbidade e mortalidade vêm aumentando nos últimos anos. Dentre os muitos argumentos levantados por pesquisadores, no sentido de se tentar explicar a elevação destas taxas, temse, de um lado, os que a associam à abordagem inadequada da asma pelos profissionais de saúde e pacientes, e, de outro, os que afirmam dever-se a: mudanças na atenção médica; mudanças no meio; e mudanças no indivíduo atópico ${ }^{(6)}$.

Esta contradição, por conseguinte, incentivou a valorização do enfoque ao paciente asmático e/ou família e/ou sobre o ambiente, como armas no combate à doença, o que tem levado ao desenvolvimento de consensos, debates e pesquisas direcionadas à abordagem educativa.

A enfermagem participa deste cenário, visto que ações educativas são imprescindíveis para o estabelecimento do cuidado à saúde, vindo este ampliar a visão biomédica e de corporeidade, buscando o indivíduo enquanto sujeito, assim como, atentar para a relevância da compreensão do ambiente que o rodeia e o homem neste contexto, buscando o estabelecimento da qualidade de vida. Para tanto, a pesquisa é uma estratégia indispensável para repensar e recriar a prática em saúde.

Para que isso aconteça é imprescindível que os resultados das pesquisas sejam divulgados e disponibilizados, diminuindo a distância entre o conhecimento produzido e a prática assistencial de enfermagem ${ }^{(7)}$.

Nesse sentido, a pesquisa bibliográfica assume eminente mérito em suas diversas finalidades, servindo de base para realização de outros estudos, assim como, fornecendo informações para a vida acadêmica ou para o desenvolvimento da prática profissional, além de gerar novas questões para investigação.

Portanto, torna-se fundamental conhecer o estado atual do conhecimento na área de enfermagem relativo à asma, para que novos conhecimentos sejam produzidos e outros aprofundados, uma vez que o sentido do saber vem do novo, do ir além.

Assim, o objetivo do estudo é analisar a freqüência das publicações sobre asma nos periódicos de enfermagem estrangeiros, presentes nos índices de referências informatizados da Coordenação de Aperfeiçoamento de Pessoal de Nível Superior - CAPES, e nacionais, indexados em bases de dados informatizadas, considerando-se a origem, o índice de referência, o periódico, ano de publicação, método utilizado, tipo de publicação e objeto de estudo.

\section{MÉTODOS}

Estudo descritivo realizado no período de janeiro a maio de 2005, cuja fonte foram os periódicos de enfermagem estrangeiros disponíveis no Portal de Periódicos da CAPES e os periódicos nacionais indexados nos índices de referência informatizados que contemplam publicações da área de enfermagem. Utilizou-se o descritor de assunto asthma para busca dos artigos nos periódicos estrangeiros e asma para os periódicos nacionais.

Cabe lembrar que a obtenção das referências bibliográficas estrangeiras foi realizada por terminal de computador de uma Instituição de Ensino Superior Federal do Estado do Ceará com acesso à internet, consultando o endereço eletrônico da CAPES, no qual são encontrados os índices SCIENCE DIRECT, BLACKWELL PUBLISHING e SAGE PUBLICATIONS.

Quanto às referências bibliográficas nacionais, estas foram obtidas por terminal de computador com acesso à internet, consultando o endereço eletrônico do Centro 
Latino-Americano e do Caribe de Informação em Ciências da Saúde (BIREME), sendo pesquisados os índices LILACS, MEDLINE e SciELO.

Ressalta-se que foram pesquisados apenas os periódicos de enfermagem indexados nas referidas bases de dados. Os dados foram coletados por meio de formulário, o qual contemplava nome do artigo, índice de referência, ano de publicação, tipo de publicação, objeto de estudo, origem, periódico e método utilizado.

Os dados estão apresentados em forma de figuras e tabelas e foram discutidos com base na literatura revisada e na experiência dos pesquisadores.

\section{RESULTADOS E DISCUSSÃO}

$\mathrm{Na}$ busca realizada foram encontrados 56 artigos estrangeiros e três artigos nacionais nos anos de 1994 a 2004.

Investigar a concepção proposta por outro(s) pesquisador (es) é um exercício de apreensão da mensagem transmitida, buscando-se uma aproximação da percepção sobre determinada temática. Portanto, torna-se óbvio que seja uma tarefa que não se cumpre simplesmente, tendo em vista que o conhecimento sofre influências histórico-sociais, e não é produzido a partir de um mero reflexo do fenômeno. Desse modo, tem-se que desvelar neste, o que lhe é constitutivo, e deve permitir que se descubra, por trás da aparência, o fenômeno tal como é realmente, trazendo para o campo científico um produto do trabalho do conhecimento humano e, portanto, um concreto pensado. Logo, faz-se necessário conhecer, entre outras coisas, o local onde a pesquisa foi publicada $^{(8)}$.

\section{Origem da publicação}

Analisando-se as publicações quanto a sua origem, pôde-se perceber, de forma parcial, a realidade mundial na qual a temática é abordada, indicando a baixa produção em periódicos nacionais, quando comparados aos estrangeiros. Do total de 59 artigos identificados, três são nacionais e 56 são estrangeiros.

A considerável discrepância entre o número de publicações sobre o tema e sua origem permite inferir, claramente, a existência de uma problemática: o tema asma, no Brasil, ainda assume condição pouco explorada no contexto da pesquisa de enfermagem, frente a sua real importância como entidade mórbida e sua prevalência. Além disso, estimula pensar a razão para a ínfima colaboração da enfermagem brasileira, a qual pode ser relacionada a duas perspectivas: ao setor saúde e ao sistema educacional.

Quanto ao setor saúde, este é regido pelo Sistema Único de Saúde, que possui, como princípios, atenção integral ao indivíduo, família e comunidade, priorizando- se as atividades preventivas [grifo nosso], sem prejuízo dos serviços assistenciais ${ }^{(9)}$. No entanto, tais ações preventivas ainda se encontram numa conjuntura limitada, que pode ser representada, entre outras coisas, por: instabilidade trabalhista dos profissionais, dentre eles o enfermeiro, o que desestimula e limita sua ação; uso indevido dos poucos programas formulados pelo Ministério da Saúde nessa vertente - com ênfase ao Programa de Saúde da Família - como instrumento de manipulação política e estruturação precária destes programas, sobrecarregando o profissional enfermeiro, o que o impossibilita de um compromisso com a proposta maior de prevenção.

Com relação ao sistema educacional brasileiro, seu processo de organização acontece, normalmente, desarticulando ensino-pesquisa-extensão, com ênfase no ensino tecnicista, e em detrimento ao compromisso formativo, que está na base das competências de saber pensar, aprender a aprender e de intervir de modo inovador e ético ${ }^{(10)}$.

Inserida em um modelo educacional tecnicista, a enfermagem tende a reproduzir ações de caráter assistencialista e pouco crítico.

No que concerne à pesquisa, esta se encontra extremamente associada à história da educação superior brasileira e das comunidades científicas que surgiram a partir dela. De acordo com a literatura(11), o confinamento da pesquisa, quase absoluto, ao ambiente acadêmico, finda por provocar deficiência na ciência e tecnologia. O Brasil ainda não conseguiu estimular as empresas privadas a investirem em conhecimento, estimulando a pesquisa científica como forma de crescimento e desenvolvimento, ao contrário dos países desenvolvidos, onde se tem investido bastante na pesquisa $^{(12)}$.

Nesse panorama, a potencialidade produtiva do conhecimento pela enfermagem caminha ante as dificuldades, fazendo com que temas relevantes levem muito tempo até assumir foco de atenção merecida no campo de estudo científico e nas políticas públicas do país.

Além disso, no Brasil são parcos os estudos sobre os índices epidemiológicos na asma de abrangência nacional e regional, como também são incipientes os gastos com a asma por parte do governo. As condições descritas, certamente traduzem-se em uma subvalorização da problemática no país.

A investigação da publicação indexada é uma investigação que não pode estar desvinculada dos índices de referência e periódicos nos quais os artigos foram encontrados.

\section{Índice de referência e periódico}

Tendo em vista a discussão a respeito dos índices, e 
lembrando o restrito número de publicações nacionais, compreendeu-se mais interessante analisar, separadamente, as estrangeiras e nacionais.

Dentre os artigos estrangeiros, 28 deles estão indexados na Blackwell Publishing, 20 na Science Direct e 8 na Sage Publications.

Considera-se então relevante, a explicitação de algumas características das bases de dados para melhor compreensão do material identificado. Blackwell Publishing apresenta o maior número de periódicos indexados e congrega, também, os periódicos com temáticas de maior amplitude, ou mais transversais, tais como: saúde, clínica, saúde mental, saúde da criança, enfermagem, cuidado e qualidade de vida. No Science Direct, por sua vez, apesar do considerável número de periódicos indexados, estes se encontram muito voltados para temas específicos e que se distanciam da asma, como por exemplo: acidente e emergência, psiquiatria, oncologia, anestesia, ortopedia, radiologia e cardiovascular. A Sage Publications possui menor número de periódicos indexados, e boa parte dos mesmos segue uma perspectiva mais restrita, como a base anterior, e que também não comporta, em primeiro momento, a asma, tal qual: psiquiatria, obstetrícia e ginecologia, oncologia, lactação.

Ao se relacionar o número total de artigos publicados no período selecionado, comparando-se com o número de artigos sobre asma nesse mesmo período, observouse que as três bases de dados apresentaram, proporcionalmente, a mesma freqüência de publicações sobre asma.

No que concerne às publicações nacionais, a base de dados LILACS representa 3\% e MEDLINE 2\%. Tais percentuais são pouco expressivos, considerando-se o contexto total. Contudo, delimitando-se a visão apenas à situação nacional, percebe-se que a primeira base concentra a maioria dos artigos, assumindo um significado que talvez possa ser atribuído à existência de maior número de periódicos indexados.

Considera-se relevante informar, que foram encontradas mais publicações contendo enfoque sobre a asma nos seguintes periódicos: Journal of Pediatric Nursing, com 10; International Journal of Nursing Studies e Journal of Advanced Nursing, com 4 cada; o Ambulatory Child Health, o Health Expectations, o Health Services Research, o Journal of Clinical Nursing, o Nursing and Health Sciences e o Public Health Nursing, com 3 cada; o Applied Nursing Research, o Australian Journal of Rural Health, o Clinical Nursing Research, o Journal of Child Health Care, o Journal of Emergency Nursing, o Journal of Evaluation in Clinical Practice e a Acta Paulista de Enfermagem, com 2 cada; e o Geriatric Nursing, o Home Health Care Management \& Practice, o International Journal of
Nursing Practice, o Journal of Family Nursing, o Journal of Human Lactation, o Journal of Nurse Midwifery, o Journal of Transcultural Nursing, o Scandinavian Journal of Caring Sciences e a Revista Brasileira de Enfermagem, com 1 cada.

O maior número de publicações sobre asma no Journal of Pediatric Nursing não traz surpresa, uma vez que o nome da publicação contempla temas relacionados com a criança, principal vítima de doenças respiratórias, entre elas a asma.

Percebe-se que as publicações nacionais não se encontram em periódicos que tratem especificamente do tema. Contudo, na enfermagem isso é respondido na medida em que são escassas as publicações específicas, sendo difícil conseguir de instituições particulares o incentivo à pesquisa e, conseqüentemente, a publicação do que se produz ${ }^{(13)}$.

Tendo sentido o impacto do tema no contexto mundial (nacional e estrangeiro), resta para um fechamento de sua contextualização a historicidade do processo, ou seja, sua distribuição por ano.

\section{Ano de publicação}

De acordo com o ano de publicação, a Figura 1 mostra que os artigos se apresentaram de modo crescente, com maior freqüência nos anos de 2002 (12 estrangeiros e 1 nacional) e 2004 (14 estrangeiros). Isto pode ser o reflexo do crescente engajamento dos enfermeiros em cursos de pós-graduação, o que, por sua vez, leva a um maior número de pesquisas realizadas e publicadas. Além disso, é importante lembrar que atualmente se tem número maior de periódicos indexados que há uma década.

O aumento gradativo de publicações abordando o tema asma talvez assuma uma condição de maior destaque, devido às mudanças ambientais que vêem ocorrendo no mundo todo. Logo, a condição crônica de exposição a poluentes torna, com o passar do tempo, mais acentuada a ocorrência de afecções alérgicas respiratórias, tal como a asma, avocando, cada vez mais, uma progressiva relevância científica.

Pode-se também associar este raciocínio à condição de vanguarda no ensino superior dos países desenvolvidos (Europa e EUA) e de contato com o método científico - surgido no renascimento Europeu $^{(14)}$. Portanto, ao se reunir todos esses fatores, percebe-se um maior amadurecimento científico e, devido sua condição de desenvolvimento, maior preocupação com a qualidade de vida da população.

Ainda no campo científico, o pesquisador deve estar atento para refletir sobre este concreto pensado (produto do trabalho do conhecimento humano) a fim de perceber criticamente, através do seu método, a qualidade da informação. 


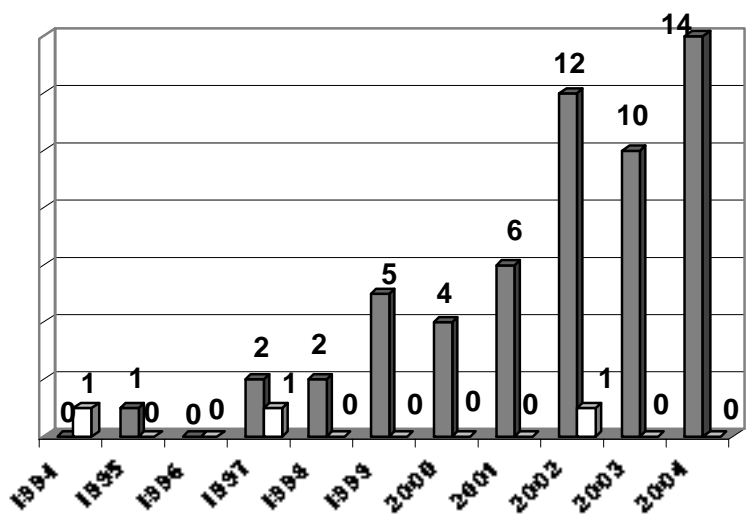

Estrangeiro $\square$ Nacional

Figura 1 - Distribuição das publicações sobre asma por ano, $2005(\mathrm{n}=59)$.

\section{Método utilizado}

Método é o conjunto das atividades sistemáticas e racionais que, com maior segurança e economia, permitem alcançar o objetivo, traçando o caminho a ser seguido, detectando erros e auxiliando as decisões do cientista ${ }^{(15)}$. Existem, basicamente, dois métodos nos quais a pesquisa pode se enquadrar: o quantitativo, apoiado pelo positivismo e neo-positivismo, e o qualitativo, que possui uma abordagem mais compreensiva. Encontrou-se, neste estudo, que 31 publicações utilizaram o método quantitativo e 28 utilizaram o método qualitativo.

A tendência atual é aceitar que não existe método certo ou errado, melhor ou pior. Do ponto de vista metodológico, não há contradição, assim como não há continuidade, entre investigação quantitativa e qualitativa. Ambas são de natureza diferente. A investigação quantitativa atua em níveis de realidade e tem como objetivo trazer à luz dados, indicadores e tendências observáveis. A investigação qualitativa, ao contrário, trabalha com valores, crenças, representações, hábitos, atitudes e opiniões ${ }^{(16)}$.

O que é defendido, atualmente, é que os dois métodos se complementam e que as experiências das pesquisas de campo, baseadas em uma perspectiva mais pragmática e menos orientada para um sectarismo epistemológico, sugerem que, da combinação das duas abordagens (cada uma no seu uso apropriado), é possível obter ótimos resultados ${ }^{(17)}$.

Entretanto, não podemos desconsiderar que são as pesquisas quantitativas que orientam os processos de tomada de decisão na planificação, formulação e aplicação de políticas públicas. Nesse sentido, cabe refletir sobre as publicações de enfermagem nacionais sobre asma, pois todos os trabalhos nacionais eram de natureza qualitativos.

\section{Tipo de publicação}

Os trabalhos encontrados foram classificados como: editoriais, artigos originais, artigos de atualização, artigos de revisão e relato de experiência.

A pesquisa mostrou que a grande maioria dos estudos encontrados (45 artigos) classifica-se como artigos originais. Este dado pode ser explicado tendo em vista que os periódicos concedem ,maior espaço para a publicação de artigos originais, visto que é este tipo de pesquisa que amplia o conhecimento científico de determinado fenômeno, desvelando novos achados, confirmando ou refutando achados de outras pesquisas e demonstrando particularidades dentro da realidade mundial.

Por outro lado, os demais tipos de publicação refletem o conhecimento já produzido por outros estudos. Nesse sentido, a importância destas pesquisas reside no fato de que a produção deste saber contribui para a reflexão da prática.

\section{Temas de estudo}

A compreensão do objeto a ser estudado é fundamental na construção da pesquisa, uma vez que esse representa a estrutura de todo trabalho científico. O objeto de estudo é que conduz o desvelar do problema de pesquisa. Este último esclarece a dificuldade específica com a qual se defronta e que pretende resolver por intermédio da pesquisa ${ }^{(18)}$.

Neste caso, o objeto dos estudos encontrados sobre asma demonstra os caminhos seguidos até o momento acerca deste tema.

A Tabela 1 nos mostra que a educação em saúde (14 estudos) é o objeto que mais estimula os pesquisadores enfermeiros no campo da asma. É sabido que, apesar do maior conhecimento dos aspectos fisiopatológicos e de tratamento da asma, a respectiva morbidade e mortalidade vêm aumentando. Isto acaba por direcionar as pesquisas dos enfermeiros para a temática "educação em saúde", visto que, pela condição crônica da doença, o paciente necessita de atividades de educação em saúde para melhor lidar com a doença.

Tabela 1 - Distribuição dos artigos sobre asma por objeto de estudo, 2005. ( $\mathrm{n}=59)$

\begin{tabular}{lrr}
\hline OBJETO DE ESTUDO & f & $\mathbf{0}$ \\
\hline Educação em saúde & 14 & 23,8 \\
Tratamento da asma & 12 & 20,5 \\
Família da criança asmática & 10 & 17,0 \\
Condições fisiopatológicas & 6 & 10,0 \\
A vida do asmático & 6 & 10,0 \\
Assistência de Enfermagem & 5 & 8,5 \\
Dados epidemiológicos sobre asma & 3 & 5,1 \\
Auto-cuidado & 2 & 3,4 \\
Asma em idosos & 1 & 1,7 \\
\hline TOTAL & 59 & 100,0 \\
\hline
\end{tabular}


A vista disso é crescente a preocupação dos currículos de enfermagem na responsabilização deste profissional pela promoção da saúde. Assim sendo, a educação em saúde torna-se um dos pilares essenciais na transformação do paciente em um sujeito ativo no seu processo de cuidado.

\section{CONSIDERAÇÕES FINAIS}

Observou-se uma discrepante predominância das publicações em periódicos estrangeiros, se comparadas aos nacionais. O Journal of Pediatric Nursing foi o que mais reuniu publicações, sendo responsável por 10 destas. A produção científica estrangeira de enfermagem sobre asma aumentou ao longo do período estudado, ao contrário da nacional, que se manteve estática. O método quantitativo foi o mais freqüente, assim como os artigos originais. O objeto mais estudado foi a educação em saúde.

Este trabalho revelou a necessidade de maior produção e/ou de maior divulgação do conhecimento produzido. Também se faz necessário um número maior de periódicos nacionais indexados, para que, desse modo, o conhecimento sobre asma na área de enfermagem seja difundido de forma mais democrática. Talvez falte a percepção do enfermeiro da importância de publicar em periódicos de enfermagem, para um fortalecimento do conhecimento científico na profissão, o que favoreceria a consolidação de grupos especialistas em determinadas áreas do conhecimento.

\section{REFERÊNCIAS}

1. Sociedade Brasileira de Pneumologia e Tisiologia. III Consenso Brasileiro no Manejo da Asma. J Pneumol. 2002; 28(Supl 1).

2. Bettencourt ARC, Oliveira MA, Fernandes ALG, Bogossian M. Educação de pacientes com asma: atuação do enfermeiro. J Pneumol. 2002;28(4):193-200.

3. Cruz AA. Asma: um grande desafio. São Paulo: Atheneu; 2004.

4. Brasil. Ministério da Saúde. DATASUS. Informações de saúde. 2005[sítio na Internet]. Brasília (DF): Ministério da Saúde; c2004. [citado 2005 Jul 12]. Disponível em: http:/ /www.datasus.gov.br/

5. Sole D, Wandalsen GF, Camelo-Nunes IC, Naspitz CK; ISAAC - Brazilian Group. Prevalence of symptoms of asthma, rhinitis, and atopic eczema among Brazilian children and adolescents identified by the International Study of Asthma and Allergies in Childhood (ISAAC) Phase 3. J Pediatr (Rio J). 2006;82(5):341-6.

6. Bosi DR, Reis AOA. A criança asmática na família: estudo de uma representação. Rev Bras Crescimento Desenv Hum. 2000;10(2):60-76.

7. Almeida AP, Kimura AF Enfermagem neonatal: análise das publicações indexadas nos índices de referências informatizados. Rev Bras Enfermagem. 2003; 56(3): 254-9.

8. Andery MA, organizadora. Para compreender a ciência: uma perspectiva histórica. 5a ed. Rio de Janeiro: Espaço e Tempo; 1994.

9. Brasil. Lei n. 8.080 de 19 de setembro de 1990. Dispõe sobre as condições para a promoção, proteção e recuperação da saúde, a organização e o funcionamento dos serviços correspondentes e dá outras providências. Diário Oficial da República Federativa do Brasil, Brasília (DF), 1990 Set 20. [citado 2005 Jul 12]. Disponível em: . http://www.cff.org.br/Legisla\%C $3 \%$ A7\%CC3\%A3o/Leis/lei_8080_90.html.

10. Demo P. Educar pela pesquisa. Campinas, SP: Autores Associados; 1996.

11. Perez JF. Pesquisa: a construção de novos paradigmas. São Paulo Perspect. 2002;16(4):30-5.

12. Trigueiro MGS. A comunidade científica, o Estado e as universidades no atual estágio do desenvolvimento científico-tecnológico. Sociologias. 2001;3(6):30-51.

13. Fundação Nacional de Desenvolvimento do Ensino Superior Particular. A pós-graduação stricto sensu nas IES particulares INFOCAPES -Bol Inform da CAPES. 2000;8(3):4-30.

14. Gaarder J. O mundo de Sofia: romance da história da filosofia. São Paulo: Companhia das Letras; 1995.

15. Turato ER. Tratado da metodologia da pesquisa clínicoqualitativa: construção teórico-epistemológica, discussão comparada e aplicação nas áreas da saúde e humanas. Petrópolis: Vozes; 2003.

16. Minayo MCS, Sanches O. Quantitativo-qualitativo: oposição ou complementaridade? Cad Saúde Pública. 1993;9(3):239-48.

17. Serapioni M. Métodos qualitativos e quantitativos na pesquisa social em saúde: algumas estratégias para a integração. Ciênc Saúde Coletiva. 2000; 5(1): 187-92.

18. Lakatos EM, Marconi MA. Fundamentos de metodologia científica. Ga ed. São Paulo: Atlas; 2005. 